ERRATUM

\title{
Stratified Hamiltonians and the Optimal Feedback Control (*).
}

\author{
Ann. Mat. Pura e Appl., IV, XXXIII (1983), pp. 51-78 \\ ȘTEFAN MIRIõ̃ (Bucharest, Romania)
}

As some statements in Lemmas 2.2, 2.3 and in the proof of Lemma 2.5 are either wrong or futile, with no effect on the rest of the paper these Lemmas should be replaced by the following:

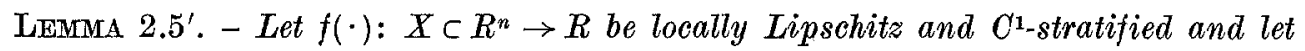
$x(\cdot):[a, b] \rightarrow X$ be absolutely continuous. Then:

$$
x^{\prime}(t) \in T_{x(t)} X \quad \text { a.e. on }[a, b]
$$

and if the derivatives of $f(\cdot)$ and $x(\cdot)$ satisfy:

$$
D f(x(t)) \cdot x^{\prime}(t)=0 \quad \text { a.e. on }[a, b]
$$

then there exists $c \in R$ such that $f(x(t))=e$ for any $t \in[a, b]$.

If the derivatives of $f(\cdot)$ and $x(\cdot)$ satisfy:

$$
D f(x(t)) \cdot x^{\prime}(t) \geqslant 0 \quad \text { a.e. on }[a, b]
$$

then $f(x(b)) \geqslant f(x(a))$

Proof. - Let $I^{d} \subset[a, b]$ be the null-set of points at which $x(\cdot)$ is not differentiable and let $I^{a}$ the finite set of the points $t \in[a, b]$ that are isolated in $x^{-1}(S)$ if $x(t) \in S \in \mathrm{S}_{f, x}$; then $I(x(\cdot))=I^{d} \cup I^{i}$ is a null-set and for any $t \in[a, b] I(x(\cdot))$ the derivative $x^{\prime}(t)$ exists and since $t \in x^{-1}(S)$ is not isolated if $x(t) \in \mathbb{S} \in \mathcal{S}_{f, X}$, there exists a sequence $t_{k} \rightarrow t, t_{k} \neq t$, such that $x\left(t_{k}\right) \in S$ and therefore

$$
x^{\prime}(t)=\lim _{k \rightarrow \infty}\left(x\left(t_{k}\right)-x(t)\right) /\left(t_{k}-t\right) \in T_{x(t)} S=T_{x(t)} X .
$$

(*) Entrato in Redazione il 15 maggio 1984. 
Further on, $g(\cdot)=f(x(\cdot))$ is absolutely continuous (since $f(\cdot)$ is locally Lipschitz) and at any $t \in[a, b] \backslash I(x(\cdot))$ at which $g(\cdot)$ is differentiable one has: $g^{\prime}(t)=$ $=D f(x(t)) \cdot x^{\prime}(t)$ (as one may see taking a local coordinate chart at $x(t) \in S \in S_{f, x}$ ); since an absolutely continuous function is the indefinite integral of its derivative, from (2.2) it follows that $g(\cdot)$ is constant and from (2.3) it follows that $g(\cdot)$ is nondecreasing and Lemma $2.5^{\prime}$ is proved. 